\title{
Non-linear finite element analysis of the dynamics of a slender cable stayed tower
}

\author{
Eliot Pezo, Paulo Gonçalves*, and Deane Roehl \\ Civil and Environmental Engineering Department, PUC-Rio, 22451-900, Rio de Janeiro, RJ, Brazil
}

\begin{abstract}
The aim of the present work is to investigate the static and dynamic nonlinear behaviour of a cable-stayed tower. A continuous structural model of a slender tower is discretized by the finite element method. First the buckling and post-buckling behaviour of the tower under axial load is explored, in order to understand the influence of the cable stiffness and lateral restrain on the load carrying capacity of the tower. Then, the linear vibration modes and frequencies are obtained. Due to the inherent symmetries of the tower, coincident buckling loads and vibration frequencies are obtained. This may lead to interactive buckling and internal resonance, increasing the effect of the geometric nonlinearities on the response. The results show that the tower exhibits a highly nonlinear response, which must be considered with care in the design stage.
\end{abstract}

\section{Introduction}

Slender guyed towers are used to support broadcasting antennas, for meteorological measurements, power transmission, etc. Nowadays, modern tall guyed towers constructed of high-strength and lightweight materials are highly flexible and lightly damped. These structures present non-linear behaviour even under working conditions [1]. The main source of nonlinearity is the change of stiffness of the guys due to the variation of stresses. Also they may exhibit undesired vibrations under environmental loads such as wind and earthquakes. Considering the strong structure sensitivity to imperfections, the structure's work conditions must follow severe requirements in terms of maximum displacements and rotations. In addition, static and dynamic instabilities and non-linear vibration of these structures are important design concerns.

\section{Phenomenological discrete model}

A 2dof simplified model of a guyed tower, supported laterally by three linear springs, as illustrated in Fig. 1, was proposed by Thompson and Gaspar [1] as an example of interactive buckling in the context of the catastrophe theory. The nonlinear static and dynamic behaviour of this model was recently explored by Orlando et al. [2] and Gavassoni et al. [3]. Their results show that the inherent symmetries and post-buckling solutions have a marked influence on the underlying potential function and, consequently, on the nonlinear dynamics of the system. The model is composed by an inverted spatial pendulum with a tip-mass $m$ and a slender, rigid (but massless) bar of a length $l$ pinned at the base, connected to three linear springs. The springs are symmetrically positioned around the tower.

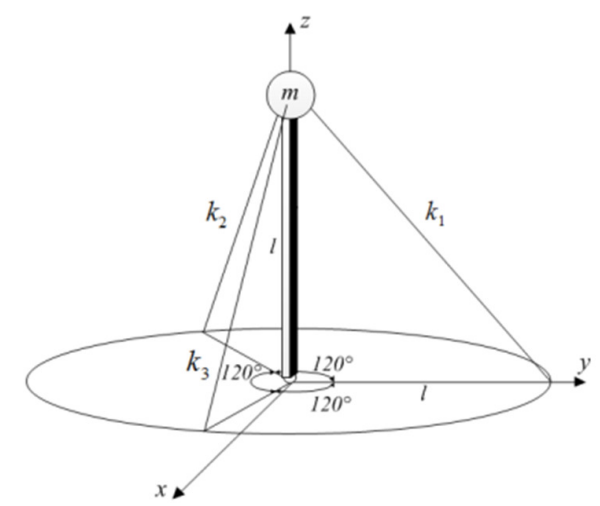

Fig. 1. 2DOF model of a guyed tower

\section{Finite element model}

In order to understand the behaviour of the continuous system and infer if it exhibits the same type and degree of nonlinearity of the discrete model, a $100 \mathrm{~m}$ tall tower clamped at the base and supported laterally by three guys inclined at $60^{\circ}$ and equally distributed around the tower is considered. The numerical analysis of the elastic guyed tower is carried out with a finite element model composed by a tower discretized with 10 two-node cubic spatial beam elements and three cables. The cables are discretized with linear three-dimensional truss elements. Figure 2 presents the geometry and finite element model of the structure.

The tower presents the following properties: cross section $0.0191 \mathrm{~m}^{2}$, inertial moment $5.69 \mathrm{E}-4 \mathrm{~m}^{4}$, and Young's modulus $1.18 \mathrm{E}+8 \mathrm{kN} / \mathrm{m}^{2}$. The cable properties

\footnotetext{
Corresponding author: paulo@ puc-rio.br
} 
are: diameter $0.018 \mathrm{~m}$, cross section $2.5446 \mathrm{E}-4 \mathrm{~m}^{2}$ and Young's modulus $1.0 \mathrm{E}+8 \mathrm{kN} / \mathrm{m}^{2}$.

An initial pretension of $10 \mathrm{kN}$ is applied to the cables defining an initial stress and deformation state. Figure 3 and Figure 4 show the guyed tower with cables loaded at $10 \mathrm{kN}$, before and after equilibrium, respectively. Unloading of stresses in the cables and the compression in the tower can be observed.
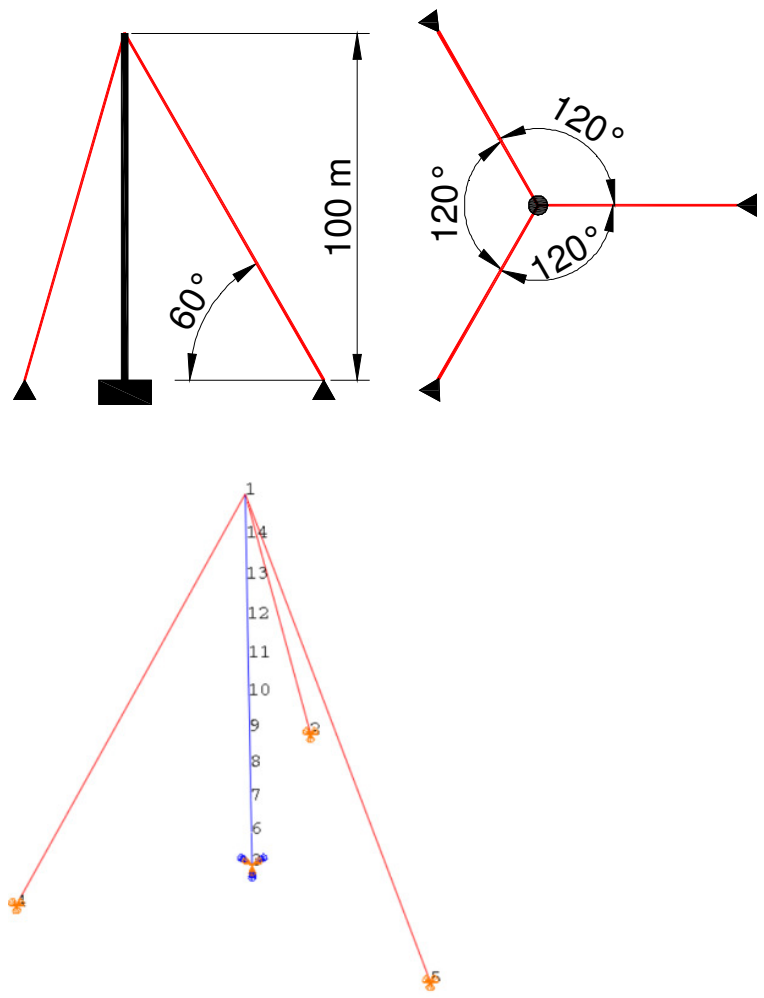

Fig. 2 Guyed tower geometry and finite element model.

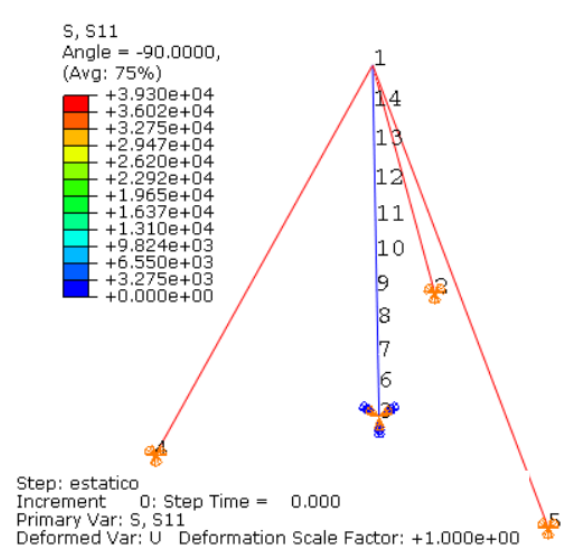

Fig. 3 Initial applied stresses- undeformed state. Stresses in $\mathrm{kPa}$.

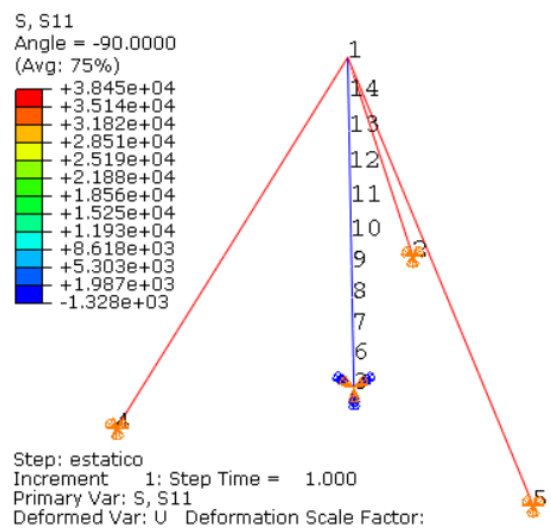

Fig. 4 Equilibrium in the initial - deformed state. Stresses in $\mathrm{kPa}$.

\section{Stability analysis - modal coupling}

\subsection{Buckling FE analysis}

Fist, the buckling loads and modes of the finite element model of the elastic guyed tower are obtained. As expected, due to the system symmetry, the first two bifurcation loads are the same, indicating the possibility of modal interaction. Table 1 provides the first four bifurcation loads for the finite element model. The buckling load of a clamped-free column with the same physical and geometrical parameters is equal to $16.6 \mathrm{kN}$. Thus, as shown in Table 1, a considerable increase in the static buckling load is observed, due to the cable stiffness, approaching the critical load of the associated clamped-pinned column. Figure 5 presents the critical loads and configurations for the first three buckling modes, where the similarity of the first two modes with the buckling mode of a clamped-pinned column is observed.
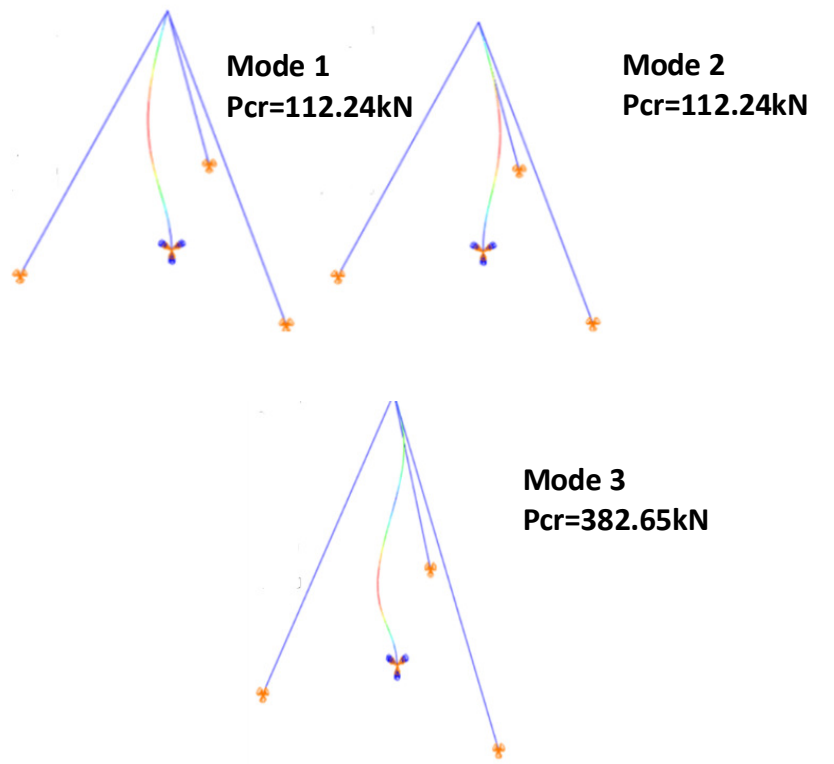

Fig. 5 Buckling modes of the finite element model 
Table 1 Bifurcation loads $(\mathrm{kN})$

\begin{tabular}{|c|c|}
\hline Mode & Pcr \\
\hline 1 & 112.24 \\
\hline 2 & 112.24 \\
\hline 3 & 382.65 \\
\hline 4 & 382.65 \\
\hline
\end{tabular}

\subsection{Post buckling FE analysis}

Next, a post buckling analysis is carried out for the tower model. The post-buckling response is shown in Figure 6, where the lateral normalized displacement components at the top of the tower are plotted as a function of the nondimensional load parameter $\mathrm{PL}^{2} / \mathrm{EI}$. The initial unstable response suggests high imperfection sensitivity, with the post-buckling minimum approaching the critical load of a clamped-free column.
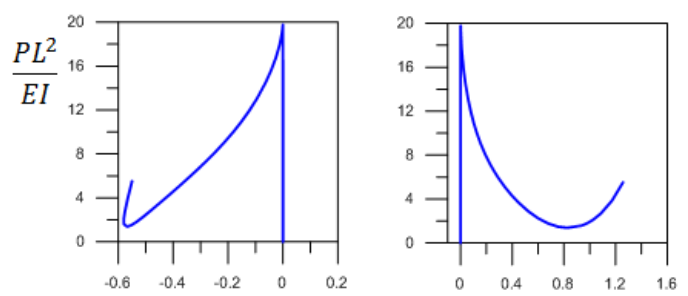

$X / L$
Fig. 6 Post critical analysis showing an unstable response

\section{Dynamic analysis}

The dynamic response is investigated for a system with a perturbation introduced through a horizontal load applied at an angle $\beta$ with respect to the $\mathrm{x}$ axis, as illustrated in Figure 7.

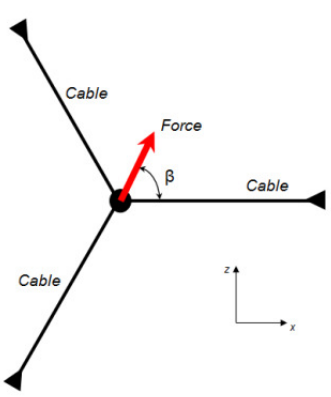

Fig. 7 Top view of the guyed tower model with load perturbation at an angle $\beta$ with the vertical plane.

The first four vibration modes and frequencies are shown in Fig. 8. Again, due to the structure' symmetries, the natural frequencies occur in pairs, which may lead to $1: 1$ internal resonance and energy transfer between these modes.

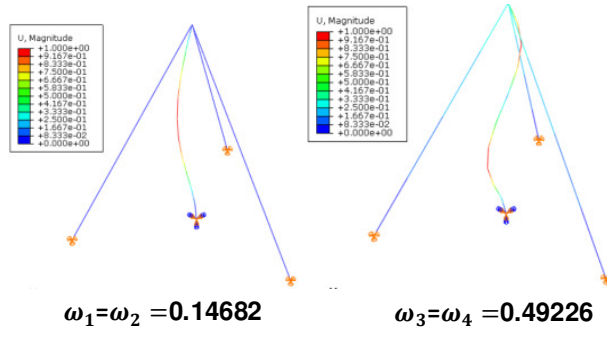

Fig. 3 Vibration modes and frequencies (Hz).

\subsection{Free vibration with initial tip perturbation}

The discrete model shows the coexisting of several similar and nonsimilar nonlinear normal modes [2, 3]. In order to investigate the existence of such modes on the continuous model, a nonlinear free vibration analysis is conducted. Figure 9 shows the nonlinear damped free vibration response considering an initial perturbation at $\beta=0^{\circ}$, while Fig. 10 shows the response for $\beta=60^{\circ}$. The initial conditions at $\mathrm{t}=0 \mathrm{~s}$ are also shown in Fig. 9. A response similar to that in Fig. 10 is obtained for $\beta=-60^{\circ}$. Thus, the same three similar nonlinear normal modes obtained for the discrete model are here obtained [2,3].
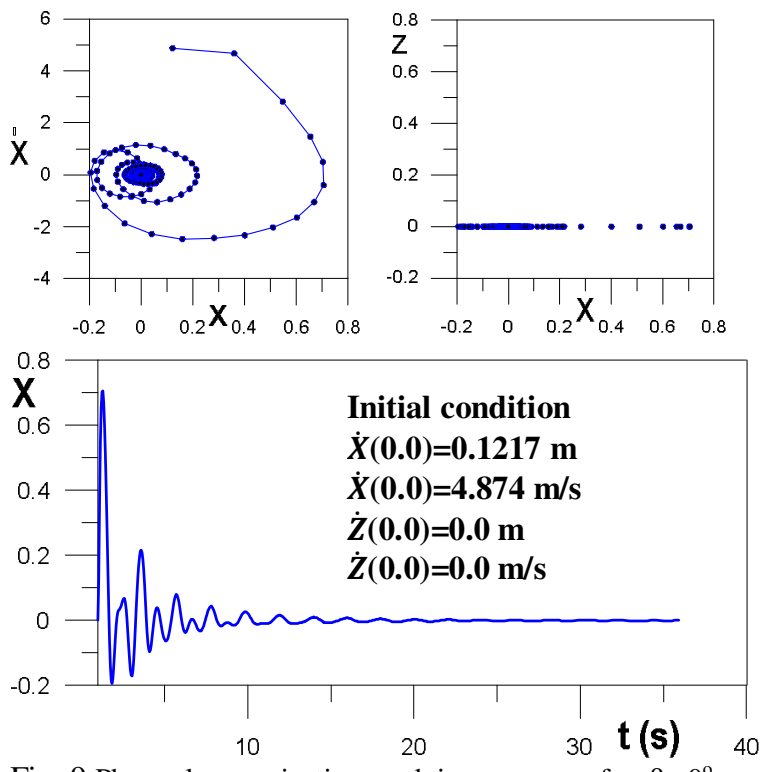

Fig. 9 Phase plane projections and time response for $\beta=0^{\circ}$.
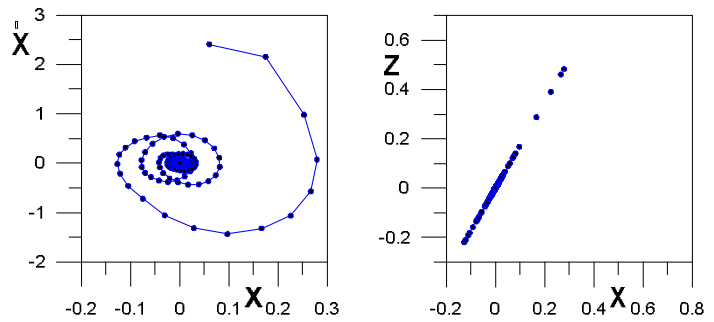

Fig. 10 Phase plane projections, displacement of top of the tower for $\beta=60^{\circ}$. 
For any other value of $\beta$ the nonlinear interaction among these NNMs is observed.

Figure 11 shows the undamped free vibration response considering again an initial perturbation at $\beta=0^{\circ}$, where energy is transferred from the $\mathrm{x}$ to the $\mathrm{z}$ direction after a high number of cycles.
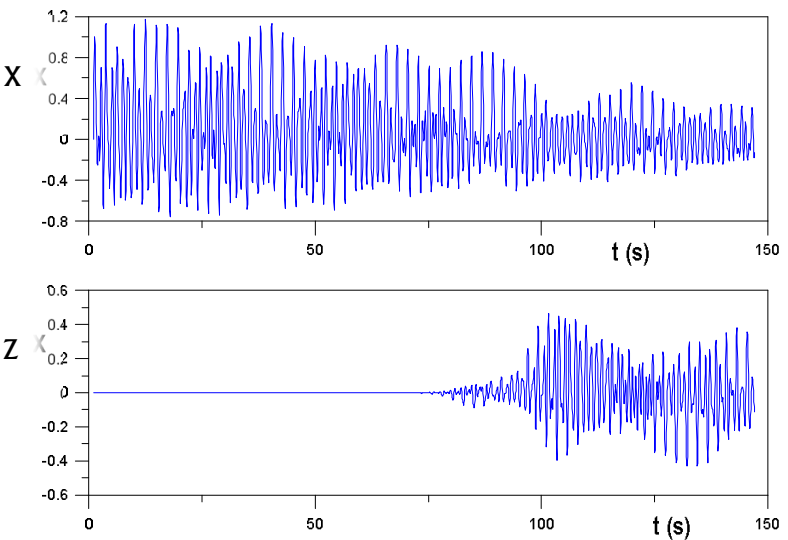

Fig. 11 Tower tip horizontal displacements in time

\subsection{Damped forced vibration}

The tower is then subjected to a distributed periodic horizontal load at an angle $\beta$, as illustrated in Figure 12 where the structure and loading are depicted.

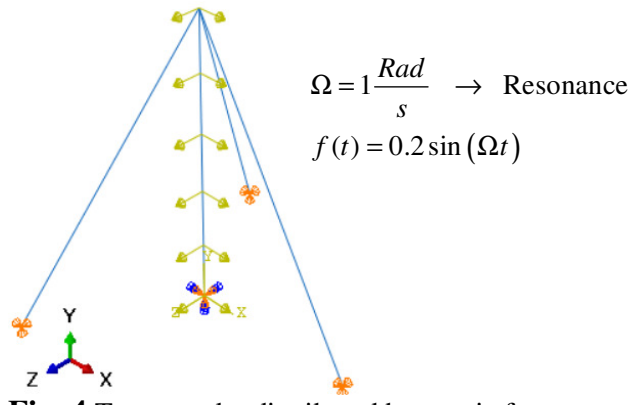

Fig. 4 Tower under distributed harmonic force
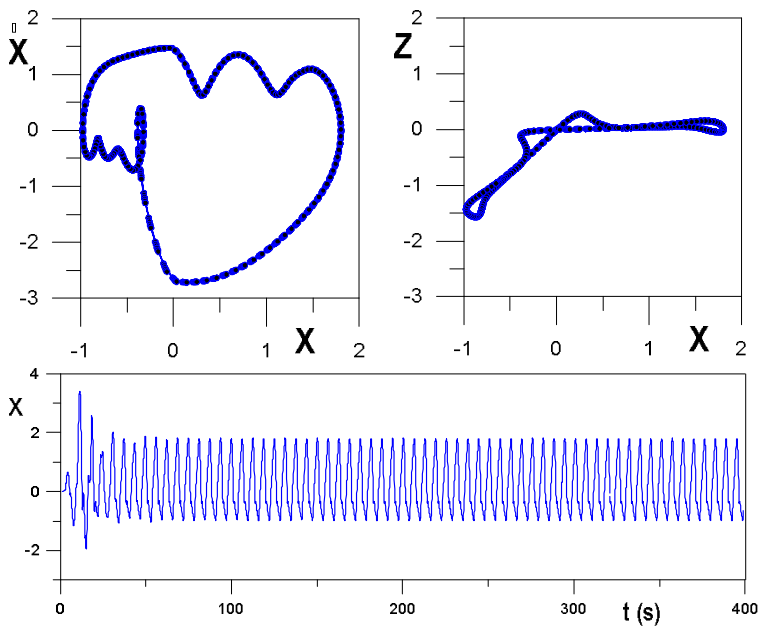

Fig. 5 Phase plane projections and time response of the tower. Inclination of the excitation force $\beta=30^{\circ}$.
Figure 13 shows two phase plane projections of the forced steady-state response and the time response of the $\mathrm{x}$ coordinate, which displays a small transient. The response in configuration space of the tower top ( $\mathrm{x}-\mathrm{Z}$ plane) show clearly the coupling between the two nonlinear normal modes depicted in Figs. 9 and 10.

\section{Final remarks}

In this work, a non-linear finite element model of a guyed tower was used to investigate the stability characteristics and nonlinear vibrations of this widely employed system. Due to their inherent symmetries, these structures display coincident or nearly coincident buckling loads leading to coupled post-buckling paths. Further, the existence of coincident and nearly coincident natural frequencies may induce even more complex behaviours with the presence of internal resonance. Again, the FE model is able to provide the expected non-linear oscillations with several nonlinear normal modes which have a fundamental importance for the understanding of the tower forced response.

\section{References}

1. M. Grey, Finite element seismic analysis of guyed mast, Master thesis, University of Oxford, Oxford, UK (2006)

2. J.M.T. Thompson, Z. Gaspar, A buckling model for the set of umbilic catastrophes, Math. Proc. Camb. Phil. Soc.. 82 (1977) 497

3. Orlando, D., Gonçalves, P.B., Rega, G., Lenci, $S$. Influence of symmetries and imperfections on the non-linear vibration modes of archetypal structural systems, International Journal of Non-Linear Mechanics Vol. 49, 175-195, 2013

4. Gavassoni, E., Gonçalves, P.B., Roehl, D. Nonlinear Vibration Modes of an Offshore Articulated Tower, Ocean Engineering, Vol. 109, pp. 226-242, 2015 